\title{
Ultrastructure of Primary Afferent Terminals and Synapses in the Rat Nucleus of the Solitary Tract: Comparison Among the Greater Superficial Petrosal, Chorda Tympani, and Glossopharyngeal Nerves
}

\author{
OLIVIA L. MAY, ${ }^{1}$ ALEV ERISIR, ${ }^{2}$ AND DAVID L. HILL ${ }^{2 *}$ \\ ${ }^{1}$ Department of Biologic and Materials Sciences, University of Michigan School of \\ Dentistry, Ann Arbor, Michigan 48109-1078 \\ ${ }^{2}$ Department of Psychology, University of Virginia, Charlottesville, Virginia 22904-4400
}

\begin{abstract}
The greater superficial petrosal (GSP), chorda tympani (CT), and glossopharyngeal (IX) nerves terminate in overlapping patterns in the brainstem in the rat nucleus of the solitary tract (NTS). There is one region, in particular, that receives overlapping inputs from all three nerves and is especially plastic during normal and experimentally altered development. To provide the requisite data necessary ultimately to delineate the circuitry in this region, we characterized the morphology of the synaptic inputs provided by the GSP, CT, and IX nerves through transmission electron microscopy. Although all three nerves had features characteristic of excitatory nerve terminals, ultrastructural analysis revealed dimorphic morphologies differentiating IX terminals from GSP and CT terminals. IX terminals had a larger area than GSP and CT terminals, and more synapses were associated with IX terminals compared with GSP and CT terminals. Additionally, IX terminals formed synapses most often with spines, as opposed to GSP and CT terminals, which formed synapses more often with dendrites. IX terminals also exhibited morphological features often associated with synaptic plasticity more often than was seen for GSP and CT terminals. These normative data form the basis for future studies of developmentally and environmentally induced plasticity in the rodent brainstem. J. Comp. Neurol. 502:1066-1078, 2007. ๑2007 Wiley-Liss, Inc.
\end{abstract}

Indexing terms: brainstem nuclei; gustatory nerves; taste; electron microscopy

The nucleus of the solitary tract (NTS) is the site of the first central synapse in the ascending gustatory system (Contreras et al., 1982). The area that receives oral sensory inputs is located in the rostral portion of the NTS and is often referred to as the "gustatory NTS" (Bradley, 2007). We recently demonstrated that there is a region in the rat gustatory NTS that receives overlapping inputs from the chorda tympani (CT) nerve, the greater superficial petrosal (GSP) nerve, and the glossopharyngeal (IX) nerve (May and Hill, 2006). These nerves innervate taste receptors on the anterior tongue, palate, and posterior tongue, respectively. In addition to providing gustatory input to the brainstem, the three nerves also supply temperature and tactile information (Frank, 1991; Nejad, 1986; Ogawa et al., 1968; Sollars and Hill, 1998). Therefore, this par- ticular region in the NTS receives a composite oralsensory input from multiple nerves and from multiple sensory modalities.

This region is also particularly interesting because it is especially plastic. In fact, the magnitude of morphological

Grant sponsor: National Institutes of Health; Grant number: DC00407; Grant number: DC06332.

*Correspondence to: Dr. David L. Hill, Department of Psychology, P.O. Box 400400, University of Virginia, Charlottesville, VA 22904.

E-mail: dh2t@virginia.edu

Received 30 June 2006; Revised 15 August 2006; Accepted 26 February 2007

DOI 10.1002/cne.21371

Published online in Wiley InterScience (www.interscience.wiley.com). 
plasticity in this zone rivals that seen in sensory cortices (Hubel et al., 1977; Killackey et al., 1978; Waite and Cragg, 1982; Wiesel and Hubel, 1965). Terminal field volumes of primary afferents change during normal development (Lasiter, 1992; Mangold and Hill, 2005; Sollars et al., 2006), in response to dietary manipulations instituted during early development (King and Hill, 1991; Krimm and Hill, 1997; May and Hill, 2006; Pittman and Contreras, 2002; Sollars and Hill, 2000; Sollars et al., 2006), and following injury to peripheral gustatory structures sustained during early postnatal ages (Lasiter and Kachele, 1990) and in adulthood (Cheon and Hill, 2003). The magnitude of these changes can be as large as $40 \times$ compared with normal adults (Mangold and Hill, 2004). Without exception, the largest effects (often the only ef-

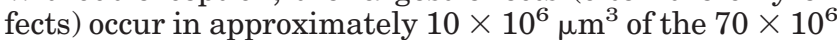
$\mu \mathrm{m}^{3}$ total terminal field. Thus, only a portion of the gustatory NTS appears to be susceptible to developmental and environmental influences.

Although terminal field labeling experiments provide important information about the organization of this particularly plastic brainstem region, characterizing the corresponding synaptic organization is essential in defining the underlying circuitry. To build upon the functional and light microscopic data (Bradley, 2007; Bradley and Grabauskas, 1998; May and Hill, 2006; Smith and Li, 2000; Smith and Scott, 2003), it is necessary to know the fundamental synaptic organization of the component primary afferents as they contact their central targets in the normal adult. This will then provide the requisite data necessary ultimately to delineate the circuitry that is so significantly altered during development and in response to experimental influences. To this end, we labeled the GSP, CT, and IX individually and examined their axon terminals, synaptic processes, and postsynaptic targets within the specific zone in the NTS where terminal fields from these three nerves overlap.

\section{MATERIALS AND METHODS}

All animal procedures were performed in accordance with NIH guidelines for humane handling of animals, and all protocols were approved by the Institutional Animal Care Committee at the University of Virginia.

\section{Animals, surgery, and tissue preparation}

Nine adult (50-60 days old), female Sprague-Dawley rats (Harlan, Indianapolis, IN) maintained on standard rat chow and tap water ad libitum were used to examine the ultrastructural morphology of GSP, CT, and IX nerve axon terminals. The GSP, CT, and IX nerves were labeled individually in separate rats, resulting in three different groups: GSP $(\mathrm{n}=3)$, CT $(\mathrm{n}=3)$, and IX $(\mathrm{n}=3)$. Animals were anesthetized with medetomadine hydrochloride (IM; $0.2 \mathrm{mg} / \mathrm{kg}$ ) followed by ketamine (IM; $20 \mathrm{mg} / \mathrm{kg}$ ) and placed on a water-circulating heating pad to maintain body temperature at $36^{\circ} \mathrm{C}$. Upon placement in a nontraumatic head holder (Erickson, 1966), a ventral approach, modified from Sollars and Hill (2000), was taken to expose either the GSP or the CT nerve in the right tympanic bulla or the IXth nerve in the ventral side of the neck medial to the tympanic bulla (May and Hill, 2006). Each nerve was cut peripheral to the ganglia. After a brief application (approximately 20 seconds) of dimethyl sulfoxide (DMSO), crystals of 3-kD biotinylated dextran amine (BDA; Molec- ular Probes, Eugene, OR) were carefully placed on the proximal cut ends of the nerves, and petroleum jelly was applied over the proximal stump to hold dye crystals in place. The incision was then sutured, and animals were injected with atipamezole (IM; $1 \mathrm{mg} / \mathrm{kg}$ ) to reverse the effects of medetomadine $\mathrm{HCl}$. The rats were allowed to recover from the anesthetic on a heating pad. As found in previous work (May and Hill, 2006; Sollars and Hill, 2000; Sollars et al., 2006), approximately 24 hours was the optimal time for anterograde transport of BDA to the NTS. Moreover, this procedure labels the full complement of myelinated and unmyelinated fibers supplied by each nerve, as evidenced by labeled ganglion cell counts (May and Hill, 2006). The method used to label each nerve does not label other nerves inadvertently, even when others are sectioned (May and Hill, 2006).

After a lethal injection of urethane ( $2 \mathrm{~g} / \mathrm{kg}$ body weight), animals were perfused transcardially for 3-4 minutes with a Krebs solution that contained $1 \%$ heparin, followed by a mixture of $4 \%$ paraformaldehyde and $1 \%$ gluteraldehyde in $0.1 \mathrm{M}$ phosphate buffer (PB; $\mathrm{pH} 7.4$ ). After postfixation in $4 \%$ paraformaldehyde overnight, the medulla was blocked and sectioned on a vibratome horizontally at $50 \mu \mathrm{m}$ through the entire NTS. Tissue sections were then reacted in a 1:100 avidin-biotinylated peroxidase complex in PBS overnight at $4^{\circ} \mathrm{C}$, followed by $1 \%$ diaminobenzidine (DAB) and $0.0003 \% \mathrm{H}_{2} \mathrm{O}_{2}$ for 5-8 minutes.

\section{Embedding}

In preparation for examination with the electron microscope, sections were postfixed with osmium tetroxide and embedded using standard procedures (Erisir and Dreusicke, 2005; Hayat, 2000). Briefly, DAB-stained sections were placed in $1 \%$ osmium tetroxide diluted in $0.1 \mathrm{M} \mathrm{PB}$ for 1 hour, dehydrated sequentially in increasing concentrations of ethanol, and stained en bloc with $4 \%$ uranyl acetate in $70 \%$ ethanol for 1 hour. Next, sections were rinsed with acetone and infused first with $50 \%$ Epon resin in acetone for 4 hours and then with full Epon overnight. Finally, the sections were flat embedded between two acetate sheets and placed in a $60^{\circ} \mathrm{C}$ oven overnight.

\section{Tissue sampling}

Projections from all three nerves terminate in the rostral, central, and medial subdivisions of the NTS (Halsell et al., 1996; Loewy and Burton, 1978; May and Hill, 2006; Whitehead, 1988; Zhang and Ashwell, 2001). We chose to focus our ultrastructural characterization of the NTS on only the dorsal zone of the composite fields, as described recently in horizontal sections by May and Hill (2006), which likely corresponds to part of the rostral central subdivision defined previously in coronal sections by Whitehead (1988) and Halsell et al. (1996; Fig. 1). Unlike other areas in the NTS, the GSP, CT, and IX nerves all provide input to this zone (May and Hill, 2006). Furthermore, the dorsal zone undergoes the most restructuring during normal development (Sollars et al., 2006) and is the most susceptible to plasticity-inducing environmental manipulations (King and Hill, 1991; May and Hill, 2006). Thus, by examining the dorsal zone alone in normal animals, we could compare how labeled axon terminals from each nerve occupy the same region with terminals from other nerves in the NTS and provide the necessary normative data for future studies that examine developmen- 

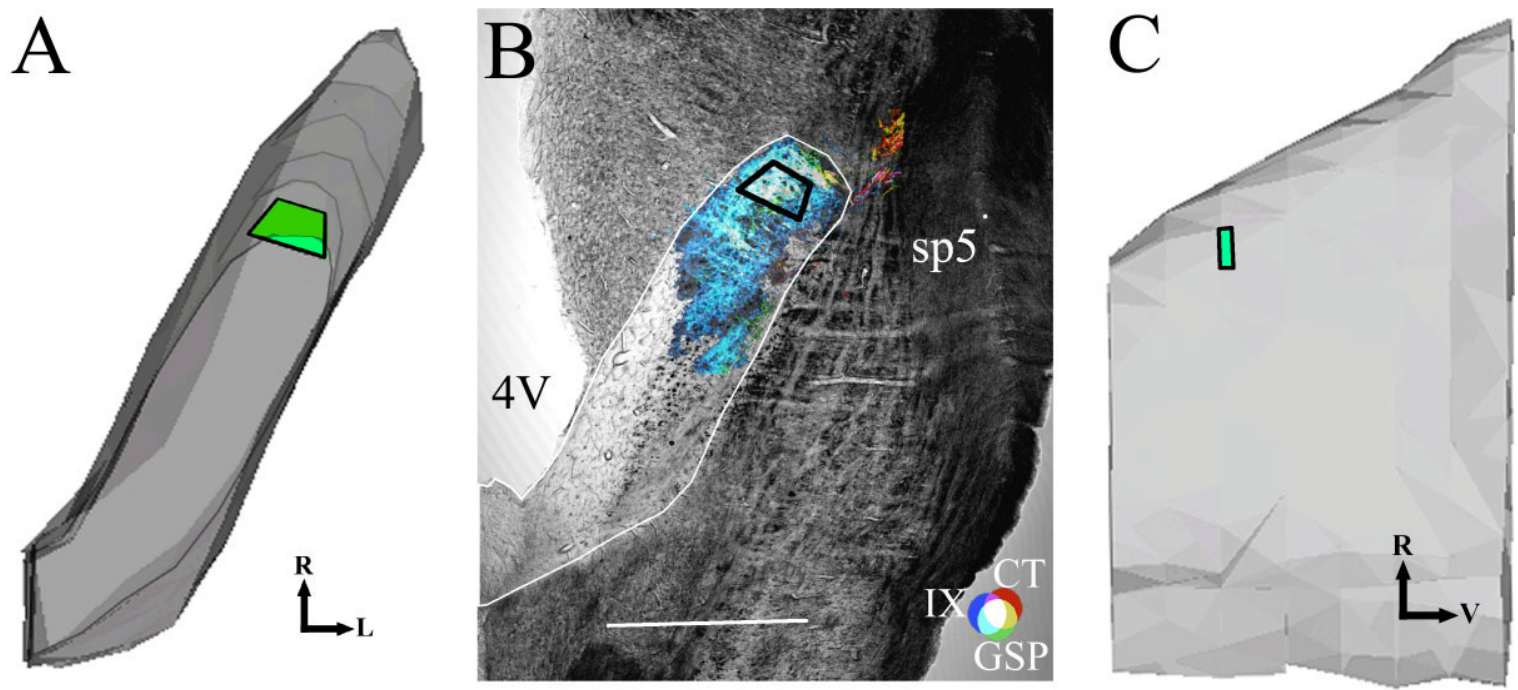

Fig. 1. A: 3D reconstruction of the right NTS (gray) viewed from the dorsal aspect. The green polygon in the rostral, dorsal portion of the NTS denotes the approximate area sampled for ultrastructural analyses. B: Photomicrograph of a representative horizontal section through the right NTS as shown in A. The colored area shows the distribution of overlapping terminal fields of the GSP, CT, and IX nerves (see color key). The polygon outlined in black denotes the approximate area in which samples were taken. The NTS is outlined in white. C: 3D reconstruction of the right NTS (gray) viewed from the lateral aspect. The green polygon in the rostral, dorsal portion of the NTS denotes the approximate area studied through electron microscopy. $\mathrm{R}$, rostral; $\mathrm{L}$, lateral; $\mathrm{V}$, ventral; $4 \mathrm{~V}$, fourth ventricle; $\mathrm{sp} 5$, spinal trigeminal tract; CT, chorda tympani; GSP, greater superficial petrosal; IX, glossopharyngeal. Orientations are the same in A and B. Scale bar $=1 \mathrm{~mm}$. tal and experimentally induced changes in synaptic organization.

Light microscopy was used to identify the dorsal zone of the NTS (May and Hill, 2006; Sollars et al., 2006). The NTS is inclined ventrally as it extends rostrally, so our term "dorsal" also refers to a dorsal/caudal location within the rostral half of the NTS (Fig. 1). To locate tissue sections that contained this zone, the outlines of the NTS, DAB-stained terminal fields, and landmarks such as the fourth ventricle; the shape of the solitary tract; and the position of the spinal trigeminal tract were drawn with a camera lucida and compared with sections and individual terminal fields characterized previously (May and Hill, 2006). The sections to be examined under the electron microscope were repolymerized on the bottom of BEEM capsules. The features of capsule-embedded sections were again drawn in detail with the aid of a camera lucida to record the appearance of the areas selected to be examined. Ultrathin sections were cut at $80 \mathrm{~nm}$ through the dorsal NTS, collected on 200 mesh copper grids, and viewed with a JEOL 1010 electron microscope. Photographs were taken with either an analog film camera or a 16-Mpixel SIA-12C digital camera (Scientific Instruments and Applications, Inc., Duluth, GA), using MaxIm DL CCD software (Diffraction Limited, Ottawa, Ontario, Canada). Photographic film was developed, digitized at 600 dpi with an Epson Perfection 1200 Photo Scanner, and examined at a final $\times 30,000-50,000$ magnification on a computer monitor.

\section{Quantitative analysis}

To document quantitative aspects of synaptic inputs to NTS, we used counting methods that correct for size through a volumetric method that we believe is best suited for the nature of the material used and for the level of reliability desired in these experiments (Guillery, 2002; Guillery and August, 2002). Counts using single ultrathin sections (i.e., size frequency/volumetric density method) were chosen over dissector approach based on the findings that, although the methods produce similar estimates of synapse density, the former is a simpler approach, allowing efficient quantification of wider brain areas in a repeated brain sample design (Colonnier and Beaulieu, 1985; DeFelipe et al., 1999). Furthermore, the use of histochemically stained sections for EM quantification provides another rationale for using single ultrathin section quantification approaches. Because of limited penetration of reagents into the thick tissue sections, the area of tissue from which the synapses are counted should be chosen within a strip of tissue (usually $10-15 \mu \mathrm{m}$ deep) that lines the tissue-Epon interface. The tissue quality of the strips at the edge of the interface can be compromised from one ultrathin section to the adjacent or near-adjacent ultrathin sections (i.e., the section used as the "look-up" section in dissector method). This introduces another level of complication to application of "assumption-free" approaches. In contrast, use of a single ultrathin section design on large tissue areas, in which the experimenter confirms the suitability, allows the sampling of relatively large number of synapses, thereby increasing the statistical power of the quantification.

Ultrathin sections were examined at the densely labeled region of the dorsal NTS. The copper grids, upon which these sections were placed, provided a means to divide sections arbitrarily into small squares of tissue. A sample area is illustrated in Figure 2A. For each selected sample, a series of overlapping pictures was taken at a relatively low magnification $(\times 1,500)$ to cover an entire grid square. 

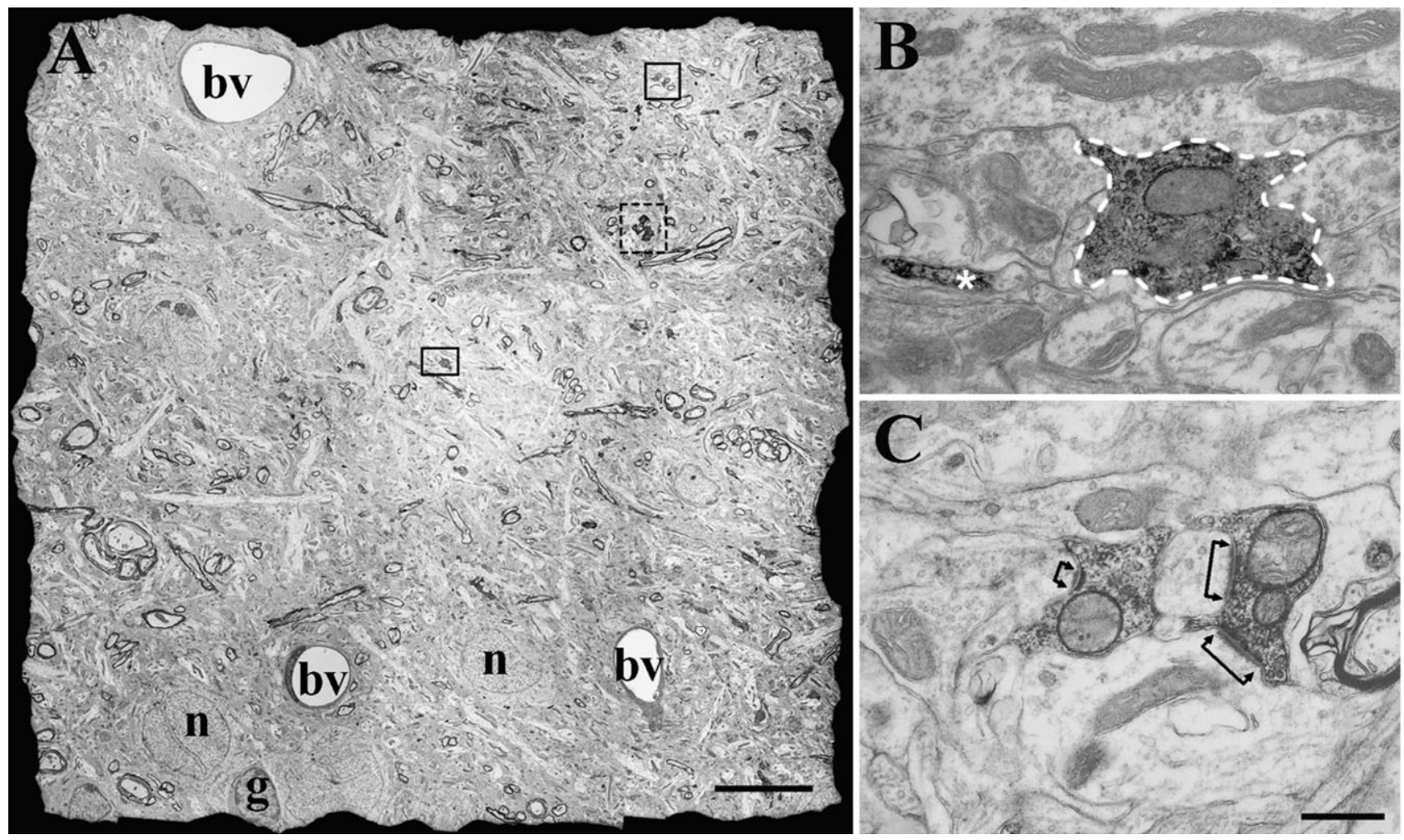

Fig. 2. A: Electron micrograph of a square division within a copper mesh grid used to examine ultrathin sections of the dorsal nucleus of the solitary tract (NTS). DAB-labeled terminals appear dark and are dispersed throughout the square of tissue. Higher magnifications of the labeled terminals that are boxed are presented in B and C. The area framed by dashed lines is shown in Figure 4B. bv, Blood vessel; g, glia; n, nucleus. B: Demonstration of a labeled axon (asterisk) and a labeled axon terminal (dashed lines), which contains synaptic vesicles. C: Two synaptic terminals making multiple synapses. Brackets mark the presynaptic membrane of each synapse. Scale bars $=10 \mu \mathrm{m}$ in $\mathrm{A} ; 0.5 \mu \mathrm{m}$ in $\mathrm{C}$ (applies to $\mathrm{B}, \mathrm{C}$ ).
In Photoshop 7.0 (Adobe Systems, San Jose, CA), a montage of these pictures was created to reconstruct each selected square of the grid. The area of the sample was computed in imaging and quantification software (Image Pro Plus, v4.5; Media Cybernetics, Silver Spring, MD) by tracing the perimeter of the square, excluding any blood vessels or Epon patches (Fig. 2A). Typically, each area constructed in this manner averaged $( \pm$ SEM) $63.0 \pm 2.0$ $\mu \mathrm{m}^{2}$.

Density data set. Each selected grid square was systematically examined with the electron microscope for the presence of DAB-labeled profiles. When a profile was encountered, a photograph was taken at $\times 10,000$. We collected images from every labeled profile within each predetermined sample area, regardless of whether the labeled profile contained a synapse. For each afferent nerve (GSP, CT, or IX), three animals were processed, and three grid squares were examined within each animal, resulting in a total of nine grid squares quantified for each nerve [GSP $(\mathrm{n}=9)$, CT $(\mathrm{n}=9)$, and IX $(\mathrm{n}=9)]$. Data collected in this manner were used for all density analyses (see below under Derived measures).

Terminal area data set. For quantifying the terminal cross-section areas of the three nerves, we used the terminals that were already photographed as the density analysis data set, with one exception. As our subsequent analysis confirmed, the labeled GSP axons displayed the lowest synapse density, yielding small numbers of synaptic terminals that were present in the density dataset. To obtain a population size comparable to that for CT and IX axons, we photographed 20 additional labeled GSP terminals. To sustain the sampling strategy, these additional labeled terminals were sampled in the same way in which we collected the density data set. These additional data were not included in any density analysis in which the area of the grid square was a variable (i.e., volumetric density of synapses and extrapolated axon length).

Identification criteria for profiles. Both labeled and unlabeled profiles were evaluated in photomicrographs taken of each labeled profile. When a profile contained DAB chromagen, it was identified as a labeled axon representing the particular nerve (Fig. 2B). If a labeled axon, or an unlabeled profile contained vesicles, it was called an axon terminal or, briefly, a terminal (Fig. 2B). The terminals that displayed a synapse were called synaptic terminals (Fig. 2C). Synapses were recognized by the existence of 1) a parallel alignment of a segment of the presynaptic membrane to the postsynaptic membrane, 2) at least three vesicles in a profile, and 3) at least one vesicle in the presynaptic profile in contact with the presynaptic membrane (Colonnier, 1968). For all identified synapses, the postsynaptic profile was classified as a spine, a dendritic shaft, an axon terminal, or a cell soma. Cell somas were identified by the presence of an endoplasmic reticulum, 
subsynaptic cisterna, a Golgi apparatus, or a nucleus. Postsynaptic axon terminals were identified by the presence of vesicles, regardless of the presence of a synapse. The direction of an axoaxonal synapse was determined such that the presynaptic element contained vesicles accumulated by the synapse and the postsynaptic element displayed a prominent postsynaptic density. Dendritic profiles displaying microtubules or mitochondria were classified as shafts. A profile was classified as a dendritic spine when neither mitochondria nor microtubules were present (Peters et al., 1970). Dendritic spines often displayed a spine apparatus, or they were observed as emerging from a dendritic shaft. However, these were not essential criteria for the identification of a spine.

Measurements. Several measurements were taken in Image Pro Plus: 1) synapse length: the length of the postsynaptic density along the parallel pre- and postsynaptic membranes of the profiles was measured; 2) area of synaptic terminals: the perimeter of each labeled terminal that contained a synapse was outlined, and the area inside the outline was calculated; 3) axon diameters: the feret minimum and feret maximum features of Image ProPlus were used to measure maximal length and width of the outlined axon (these measurements represented the largest and the smallest caliber of the labeled profile); 4) postsynaptic profile diameter: the perimeters of the postsynaptic profiles of the labeled synaptic terminals were outlined, and the largest caliber was computed.

\section{Derived measures of synaptic influence of GSP, CT, and IX nerves on the rostral NTS}

To provide an estimate of frequency of synapses provided by each nerve within a unit volume of NTS, we performed three calculations. First, we estimated how dense the labeled axons were in the NTS (areal axon density) by using a calculation for length of axon segment that each labeled profile represented, as described below. Second, we calculated the frequency of synapse formation along each axon (synapsing frequency); this gives an estimate for the number of synapses formed by a unit length of axon. Third, we calculated the volumetric density of labeled synapses. This is a measure of how many synapses each afferent nerve provides into a unit volume of the NTS. It reflects the number of synapses for each single fiber. These three derived measures were obtained as follows.

Areal axon density. To estimate how dense the labeled axons were, we first had to calculate the length of axon segment that was represented by each labeled profile we observed. For this, we used a previously published approach (Erisir and Dreusicke, 2005). Briefly, the smallest caliber $\left(\mathrm{C}_{\mathrm{s}}\right)$ and largest caliber $\left(\mathrm{C}_{\mathrm{l}}\right)$ of a profile as well as the section thickness (t) were applied in the formula below to estimate the length of the axon segment that each labeled profile represented.

$$
\text { Estimated axon length }(\mathrm{mm})=\sum\left(\sqrt{C_{l}^{2}-C_{s}^{2}}\right)+t
$$

Then, the total length of axon measured was calculated by summing the estimated length of each labeled profile in each sample grid square of tissue. An estimate of the axon density within a given area was derived by dividing the total estimated axon length by the measured area of the grid square, which contained all the labeled profiles observed for each case. Note that this is an estimate of how densely axons occupy a given area in the brain, which is comparable to estimating the axon density that can be calculated at the light microscopic level with single thick sections. The advantage of using electron microscopy for this analysis is the added precision of area measurements that excludes the areas of capillaries and cell bodies.

Synapsing frequency. To estimate the frequency of synapses along labeled axons, we divided the number of synapses observed by the total estimated length of all labeled axons for each grid. The outcome is reported as number of synapses per millimeter of axon. Note that this quantification is comparable to bouton counts along axon lengths measured via light microscope, with the added precision of confirmed synapses.

Volumetric density of synapses. To calculate the volumetric density $\left(\mathrm{N}_{\mathrm{V}}\right)$ of labeled synapses, we applied a previously published quantification approach (Colonnier and Beaulieu, 1985; DeFelipe et al., 1999), using the formula $\mathrm{N}_{\mathrm{V}}=\mathrm{N}_{\mathrm{A}} / \mathrm{d}$ (d is the average synapse length, and $\mathrm{N}_{\mathrm{A}}$ is the number of labeled synapses per unit area). The synapse length in this quantification approach provides a normalization factor for the sampling bias introduced by the thickness of the tissue, which is less than the size of the counting unit (i.e., the length of the synaptic zone). Furthermore, Colonnier and Beaulieu (1985) and DeFelipe et al. (1999) demonstrated that the validity of this quantification approach is comparable to stereological approaches and can be used reliably in estimating the number of synapses contained within a unit volume.

\section{Photomicrograph production}

Electron photomicrographs were produced by digitizing negative films at a resolution of 1,600 dpi to yield enlargements at 400-600 dpi without resampling the image. In addition, images captured using a 16-Mbyte digital camera were displayed at $600 \mathrm{dpi}$ and resampled to reduce the size of the electron micrograph to the same magnification as the digitized negatives. Photoshop 7.0 was used to compose and label the plates. Minimal brightness and contrast adjustments were performed.

\section{Methodological considerations}

There are key methodological considerations that must be addressed in interpretation of our ultrastructural data. For example, we chose to study female rats in this study because females were used in all of our other terminal field experiments (King and Hill, 1991; Krimm and Hill, 1997; May and Hill, 2006; Sollars and Hill, 2000). We would expect that significant within-group variances would be apparent for these measures if factors such as hormonal levels had effects on pre- and postsynaptic elements. This did not occur (see under Data comparisons among animals within each group in Results). Furthermore, there is no direct evidence that NTS neurons are as sensitive as hippocampal neurons to the effects of estrogen on spine and synapse formation (Gould et al., 1990; Woolley et al., 1996, 1997), nor is there convincing evidence of sex-related functional differences in NTS function. Moreover, NTS neurons do not have a complex spine component to their morphologies (King and Hill, 1993; Lasiter et al., 1989).

Because of the small diameter and location of the nerves, it is necessary to section the afferent nerve before applying anterograde tracers. Indeed, this is the only technique used previously to examine terminal fields and the ultrastructural characteristics of primary afferent fibers 
in the NTS (Brining and Smith, 1996; Contreras et al., 1982; Davis, 1998; King and Hill, 1991; May and Hill, 2006; Whitehead, 1986, 1993). However, in no instance did we find evidence of degenerating terminals and axons, such as presence of vacuoles or mitochondria condensation (Erb and Povlishock, 1991; Gillingwater et al., 2006; Somogyi et al., 1981). Moreover, we showed previously that the effects of CT axotomy on terminal field degeneration were not noticeable until after 7 days postsection (Cheon and Hill, 2003). Others have also failed to show significant ultrastructural evidence of degenerating synapses within 1 day postinjury. For example, there was an absence of degenerating profiles in the cortex of mice sustaining nearby and extensive cortical damage until after 1 day postlesion (Gillingwater et al., 2006).

\section{Statistical analysis}

Statistical comparisons for all measures were performed by ANOVA. Post hoc comparisons among GSP, CT, and IX means were accomplished with Bonferonni posttests, unless variances were not homogeneous (i.e., determined by Levene statistic). The variances were not homogeneous for tests of synapse frequency, areal axon density, and axon length. For these three tests, post hoc comparisons were performed with Dunnett's T3 test (SPSS). The alpha level of 0.05 was used to determine statistical significance. Post hoc probabilities are reported in the text.

\section{RESULTS \\ Data comparisons among animals within each group}

Data from the three grids taken from each rat were pooled and then compared with similar data from rats within the same nerve-labeled group (GSP, CT, IX). Such a statistical comparison allowed an assessment of within-group variation. We tested synaptic length, synaptic terminal area, and percentage of synapses made on dendrites, spines, and axons. There were no within-group differences for any of the measures ( $P$ values for ANOVAs $>0.30)$.

\section{Qualitative properties of labeled terminals}

At the light microscopic level, each nerve label resulted in a dense network of DAB-labeled fibers in the dorsal portion of the rostral NTS (Fig. 3A). The termination patterns of each nerve were similar to the fluorescentlabeled terminal fields characterized in May and Hill (2006). Each afferent displayed varicosities (presumed terminal boutons) along the axons like beads on a string. Terminal swellings at the ends of axon branches were also observed (Fig. 3B). However, finer details regarding those boutons were better revealed at the ultrastructural level.

At the electron microscopic level, DAB-labeled profiles appeared dark and densely filled. The intensity of the label did not obscure cellular membranes, postsynaptic densities, vesicles, or organelles, allowing qualitative and quantitative evaluation of intracellular organelles.

\section{Quantitative analysis of afferent axon terminals}

Terminal area. Preterminal axons from the GSP, CT, and IX nerves were generally unmyelinated, and axon terminals of these afferents contained round clear vesicles and dark mitochondria (Fig. 4A-C). The distribution of
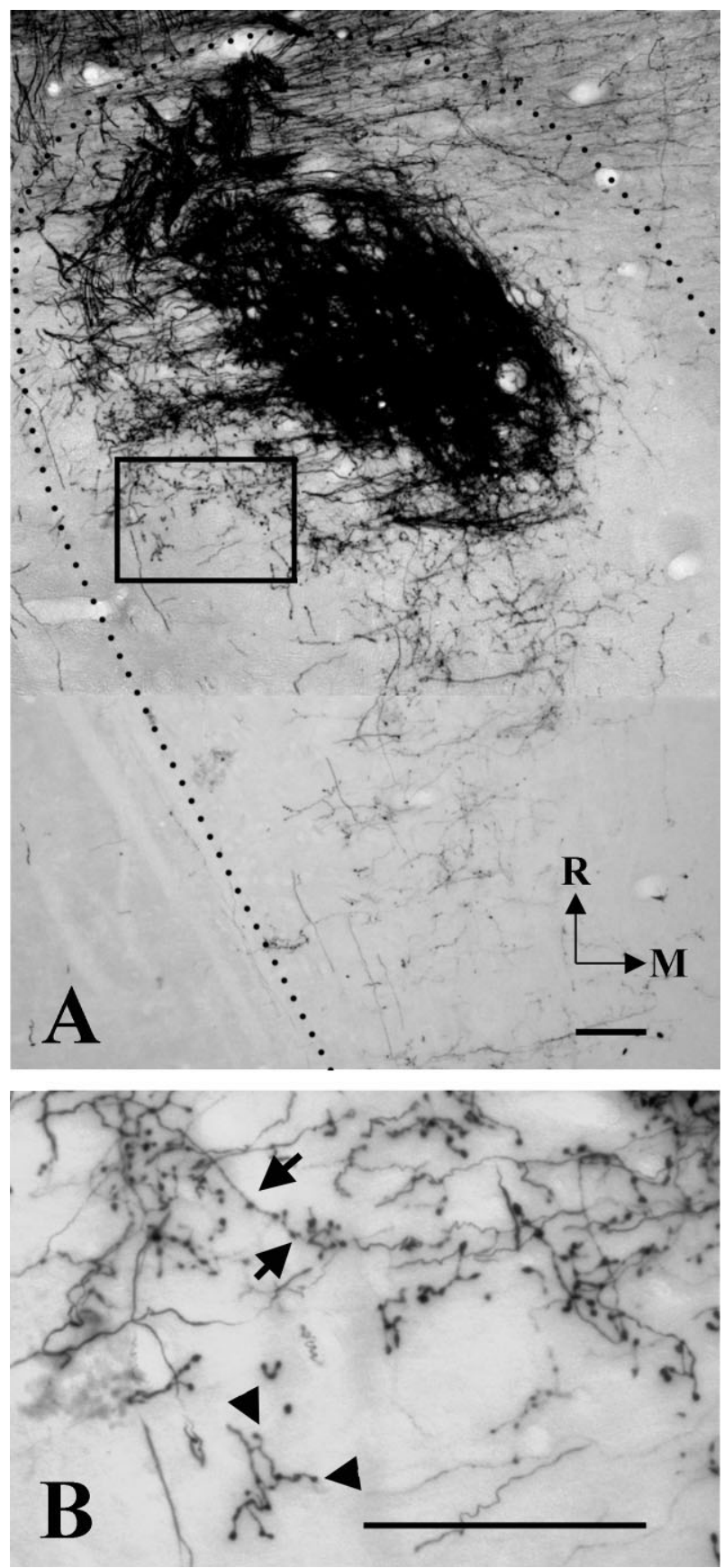

Fig. 3. Light micrographs of DAB-labeled chorda tympani (CT) nerve terminal field in the dorsal NTS of an adult rat. A: A horizontal section through the brainstem displaying the densely labeled terminal field in the dorsal NTS. The border of the NTS is outlined (dotted line). The boxed area is magnified in B. R, rostral; M, medial. B: Higher magnification of the periphery of the dense network of terminal field reveals individual fibers displaying frequent swellings (arrows). Terminal boutons are apparent as well (arrowheads). Scale bars $=10 \mu \mathrm{m}$.

the area of GSP, CT, and IX axons revealed significant size differences in the terminal endings of IX axons compared with the terminal endings of GSP and CT axons (Fig. 5A). 

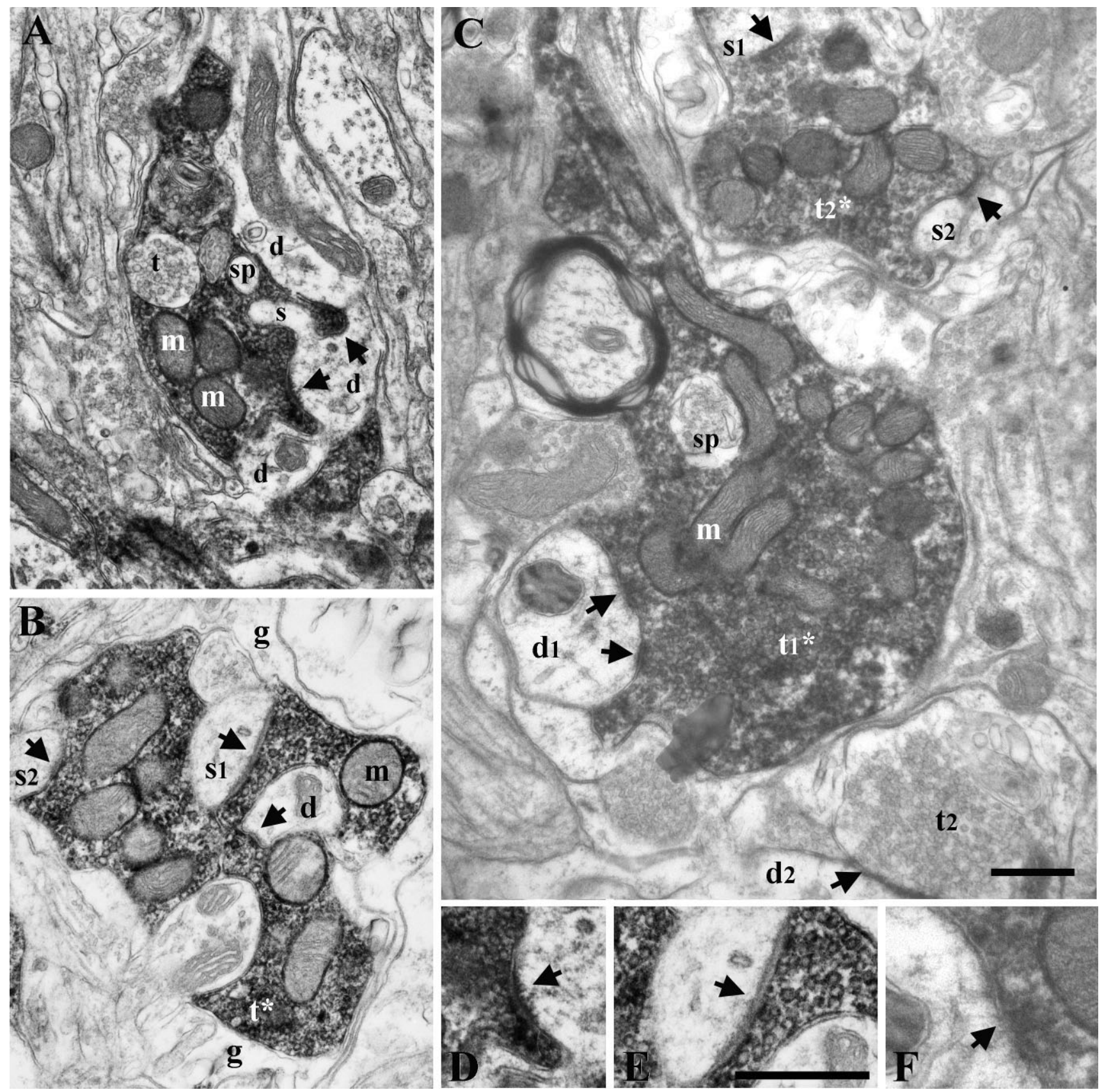

Fig. 4. Electron micrographs of DAB-labeled, vesicle-filled terminals in the dorsal zone of the NTS. A: A labeled GSP terminal forms a perforated synapse (arrows) on a dendrite (d). A spine (s) protrudes from the dendrite at the site of the synapse and into the terminal. Some unlabeled inclusions observed in labeled profiles were assumed to be cross-sections of protruding spines and, hence, were labeled spinules (sp). Other unlabeled inclusions were identified as axon segments $(\mathrm{t})$ when they contained synaptic vesicles. $\mathrm{m}$, Mitochondria. B: A labeled CT terminal ( $\left.t^{*}\right)$ is forming multiple synapses (arrows) on both a dendrite (d) and spines (s1, s2), some of which are protruding into the terminal. Glia (g) surrounds this structure. C: Two separate

labeled IX terminals $(\mathrm{t} 1 *, \mathrm{t} 2 *)$ are displayed. Terminal $\mathrm{t} 1 *$ contains a membrane-bound inclusion (sp) and forms a perforated synapse (arrows) on a dendrite (d1). Terminal t $2 *$ forms synapses on two separate spines (s1, s2). Nearby, an unlabeled terminal (t2) forms a synapse with a dendrite (d2; arrow). D: Increased magnification of a synapse made by the GSP terminal in A (arrow). E: Increased magnification of the synapse made by the CT terminal in B (arrow). F: Increased magnification of the synapse made by the IX terminal in $\mathrm{C}$ (arrow). Scale bars $=0.5 \mu \mathrm{m}$ in $\mathrm{C}$ (applies to $\mathrm{A}-\mathrm{C}$ ); $0.5 \mu \mathrm{m}$ in $\mathrm{E}$ (applies to D-F).

The average size of GSP and CT axon terminals was not significantly different $(P=0.5$; Fig. $5 \mathrm{~B})$. The mean area $\left( \pm\right.$ SEM) of GSP terminals was $0.9 \pm 0.1 \mu \mathrm{m}^{2}$ ranging from

0.1 to $3.3 \mu \mathrm{m}^{2}$, and the mean area $( \pm \mathrm{SEM})$ of CT terminals was $1.0 \pm 0.1 \mu \mathrm{m}^{2}$ ranging from 0.1 to $4.4 \mu \mathrm{m}^{2}$. However, IX terminals were significantly larger than both 

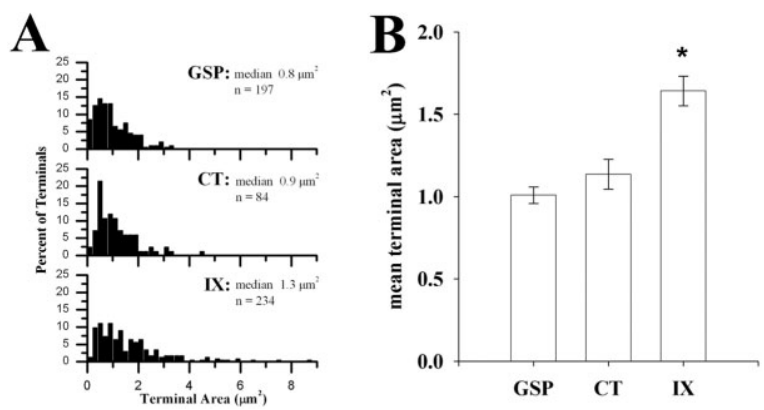

Fig. 5. A: Frequency distribution histogram of the terminal areas of DAB-labeled GSP, CT, and IX axons, measured on terminal crosssections that contained a synapse. Frequencies for each nerve are displayed as a percentage of the total to allow visual comparison among groups. IX axon terminals were significantly larger than GSP and CT terminals. Median and population size (n) are indicated for each nerve. B: Mean terminal areas of DAB-labeled GSP, CT, and IX axons. Error bars indicate standard error. ${ }^{*} P<0.05$.

GSP $(P=0.0001)$ and CT terminals $(P=0.0001$; Fig. 5B). The mean area $\left( \pm\right.$ SEM) of IX terminals was $1.6 \pm 0.1 \mu \mathrm{m}^{2}$ ranging from 0.2 to $7.6 \mu \mathrm{m}^{2}$.

Synapse length. The extent of the active zone provides one indication of the efficacy of the synaptic contacts associated with axon terminals (Pierce and Lewin, 1994). To quantify the size of the active zone, synapse length was measured. All synapses formed by labeled terminals were asymmetric (i.e., displaying a thicker density on the postsynaptic side), corresponding to Gray's type I excitatory classification (Peters et al., 1970; Fig. 4D-F). The synaptic lengths of GSP, CT, and IX terminals ranged from 0.1 to $1.0 \mu \mathrm{m}$ and had means of $0.3 \pm 0.01 \mu \mathrm{m}$ for both GSP and IX terminals and $0.4 \pm 0.02 \mu \mathrm{m}$ for CT terminals. There were no significant differences among means $(P>0.2)$.

Postsynaptic targets. All terminals formed synapses onto dendrites, spines, or vesicle containing profiles. Examples of a single terminal bouton forming multiple synapses on a single target were observed frequently for all afferent projections (e.g., Figs. 4A,C, 6A) or in more complex patterns in which an axon terminal formed synapses upon multiple postsynaptic targets (e.g., Figs. 4B, 6B). No afferent terminal formed synapses directly with cell soma in this study. Labeled terminals often appeared in glomerular arrangements in which glial membranes enveloped a collection of profiles (Fig. 6). Although infrequent, afferent terminals also engaged in triadic arrangements in which a labeled terminal formed a synapse with a target, which in turn made a synapse with another profile that was also postsynaptic to the original labeled terminal (Fig. 7). Configurations such as these were typically enclosed in glia.

To determine differences in the postsynaptic targets of GSP, CT, and IX terminals, the percentage of total number of synapses that occurred on dendrites, spines, axons, and cell somas was assessed for each labeled afferent terminal. For GSP terminals, 79\% (62/78) of the total number of synapses occurred on dendrites, and 18\% (14/ 78 ) of synapses occurred on spines. The remaining $3 \%$ $(2 / 78)$ of synapses occurred on unlabeled axon terminals. Similarly, for CT terminals, 77\% (82/107) of synapses occurred on dendrites, $16 \%$ (17/107) of synapses occurred on spines, and $7 \%(8 / 107)$ of synapses occurred on axons.
There were no significant differences in the target preferences of GSP and CT afferents; both formed synapses most often on dendritic shafts $(P=0.9)$. However, there was a significant nerve-related difference in the postsynaptic targets of IX terminals. Specifically, 35\% (121/345) of synapses from IX terminals occurred on dendrites, and $56 \%$ (194/345) of synapses from IX terminals occurred on spines. The remaining 9\% (30/345) of synapses occurred on axons. Thus, IX terminals formed synapses significantly more often with spines than with dendrites compared with that of GSP terminals $(P=0.0001)$ and CT terminals $(P=0.0001)$.

The area of tissue from which the data set for synapse analyses was derived contained few instances of cell body segments, and those that were present did not receive synapses from labeled GSP, CT, and IX afferents. To investigate the prevalence of axosomatic contacts formed by each afferent in a larger cell body population, we examined 40 neuronal cell bodies located within the projection site of each afferent. As found in the smaller sample, no labeled terminal synapsed on soma. Labeled IX axons were encountered in close proximity of the somata; however, synaptic contacts were not present.

To assess further the differences in the postsynaptic target of these afferent terminals, the relative caliber of the postsynaptic dendrites was measured. This measurement served as an estimate of the distance between where the axodendritic synapse occurred and the soma (Erisir and Dreusicke, 2005). That is, we assumed that dendritic shafts are conical, tapering away from the soma; the closer a dendrite was to the soma, the larger in diameter it would appear. The calibers of the dendrites that were postsynaptic to GSP, CT, or IX synapses were compared, and no significant differences were found $(P>0.2$; mean diameter $\pm \mathrm{SEM}-\mathrm{GSP}=1.1 \pm 0.1 \mu \mathrm{m} ; \mathrm{CT}=1.2 \pm 0.1 \mu \mathrm{m} ; \mathrm{IX}=$ $0.9 \pm 0.04 \mu \mathrm{m})$.

Terminal inclusions. A prevalent feature of labeled afferent terminals from each nerve was the presence of portions of unlabeled membrane-bound profiles within the labeled terminal. Membrane-bound inclusions appeared enclosed within labeled terminals, creating a holepunched appearance of the terminals (Figs. 4A,C, 7). Occasionally, these inclusions emerged as protrusions of postsynaptic profiles (Fig. 3A). Vesicle-filled unlabeled profiles were also included inside labeled axon terminals (Fig. 4A). Because of their similarity in definition to structures described in hippocampus and cortex (Erisir and Dreusicke, 2005; Tarrant and Routtenberg, 1977), we identified these inclusions as "spinules."

In NTS neuropil, unlabeled inclusions were observed inside GSP, CT, and IX terminals, but they occurred significantly more frequently in IX terminals. On average, $7.3 \% \pm 1.5 \%(84 / 1150)$ of labeled IX terminals observed within the dorsal NTS were invaginated by spinules. This is a significant increase in incidence compared with the less frequent occurrence of spinules inside CT terminals $(P=0.003) ; 2.0 \% \pm 0.5 \%(10 / 492)$ of CT terminals contained unlabeled patches. GSP terminals also contained fewer spinules than IX terminals $(P=0.02) ; 3.4 \% \pm 1.0 \%$ (16/473) of GSP terminals contained spinules. The difference in the frequency of unlabeled patches observed in CT and GSP terminals was not significant $(P=0.8)$

Density of axons. To gain perspective on the density of axons present in the dorsal NTS, the total axon length was expressed relative to the area of the NTS region 


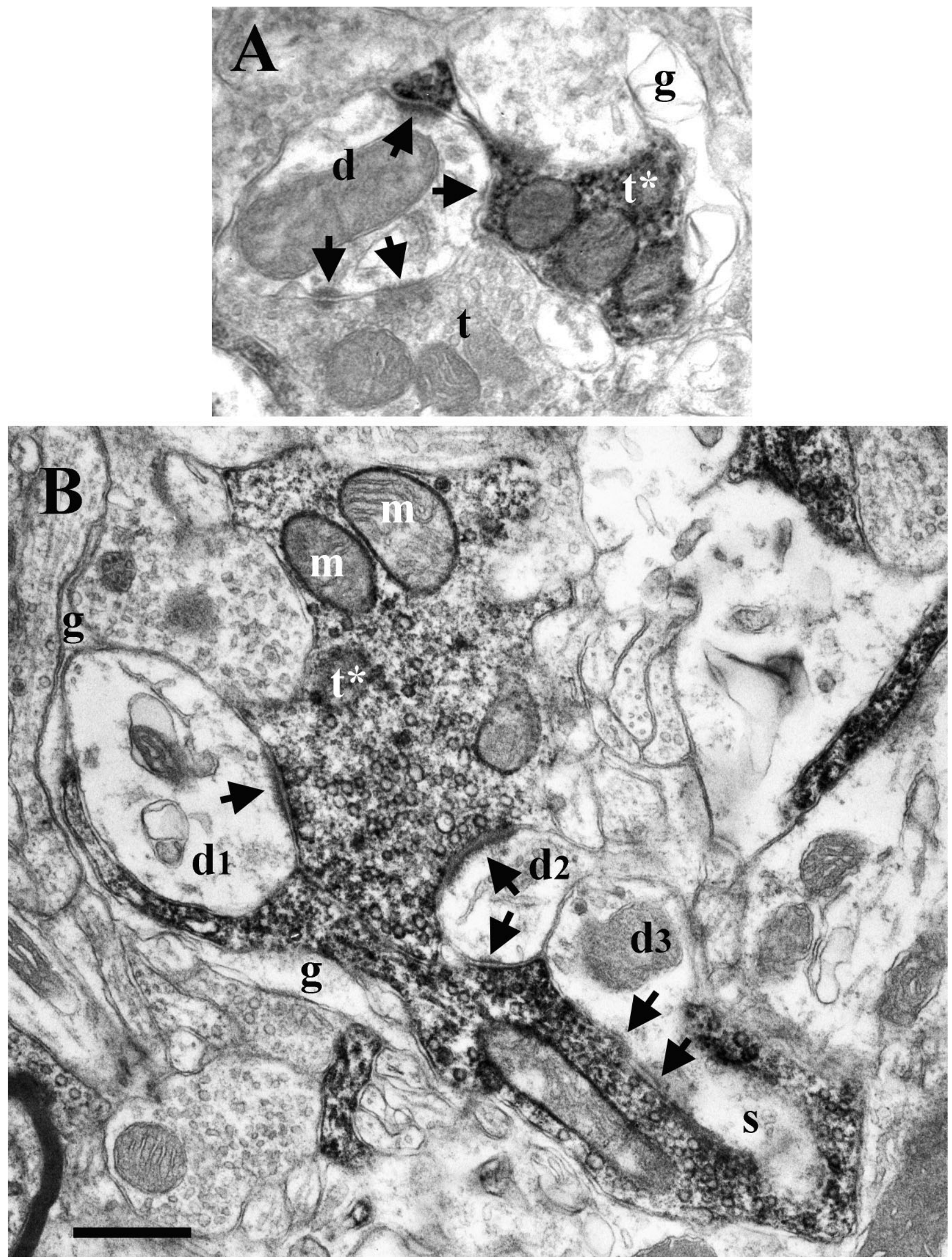

Fig. 6. A: A labeled GSP terminal ( $t *)$ and an unlabeled terminal (t) both form perforated synapses (arrows) on a dendrite. Surrounding glia (g) suggests a glomerular structure. B: A large labeled CT terminal ( $\left.t^{*}\right)$ in a glomerular formation forms synapses (arrows) with three dendrites (d1, d2, d3) and a spine (s) protruding from a dendrite (d3). Synapses on $\mathrm{d} 2$ and $\mathrm{d} 3$ are perforated. The structure is surrounded by glia (g). Scale bar $=0.5 \mu \mathrm{m}$. 


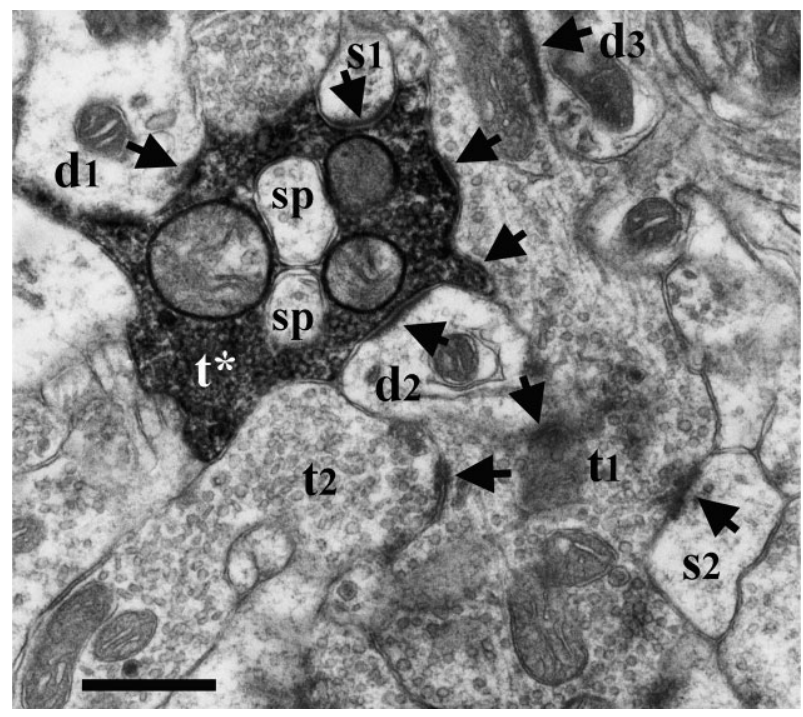

Fig. 7. A labeled CT terminal ( $\left.\mathrm{t}^{*}\right)$ that contains two spinules ( $\mathrm{sp}$ ) and forms synapses (arrows) onto two dendrites (d1, d2), a spine (s1), along with an unlabeled axon terminal (t1). The unlabeled terminal (t1) forms asymmetric synapses with a spine (s2) and a dendrite (d3), and it receives a synapse from an unlabeled terminal (t2). Thus, a triad arrangement is formed. Scale bar $=0.5 \mu \mathrm{m}$.

examined. No significant differences were found between the average areal axon densities of labeled GSP and CT axons in the dorsal NTS $\left(13.2 \pm 1.6 \mathrm{~mm} / \mathrm{mm}^{2}\right.$ for GSP vs. $12.5 \pm 1.9 \mathrm{~mm} / \mathrm{mm}^{2}$ for CT; $P=0.9$; Fig. 8A). However, labeled IX axons were significantly denser than GSP axons $(P=0.02)$ and CT axons $(P=0.02)$. IX axons had an average areal density ( \pm SEM) of $40.3 \pm 7.6 \mathrm{~mm} / \mathrm{mm}^{2}$ (Fig. 8A).

Frequency of synapses along axons. Previously, our confocal analysis revealed that GSP, CT, and IX afferents display various distributions of axonal terminations in the NTS (May and Hill, 2006). To help clarify the potential synaptic influence of these afferents in the NTS region of interest, the frequency at which the axons of GSP, CT, and IX terminals formed synapses was determined by calculating the frequency of a synapse that appeared on an axon segment.

Synapses occurred significantly more frequently with IX axons than with GSP axons $(P=0.01)$ but did not differ from CT axons $(P=0.1)$. The frequency of synapses on IX axons occurred at a rate of $175.1 \pm 22.1$ synapses $/ \mathrm{mm}$ of axon. Synapses occurred less frequently on CT axons, at a rate of $132.8 \pm 18.8$ synapses $/ \mathrm{mm}$ of axon. Additionally, synapses occurred on GSP axons at a rate of $104.8 \pm 12.7$ synapses/mm of axon (Fig. 8B).

Volumetric density of synapses. The relative density of synapses of labeled axons contained within a measured volume of the dorsal NTS was also compared among GSP, CT, and IX afferents. For unit volume of the NTS region examined, there was a significantly higher density of synapses associated with IX axons compared with the density of synapses associated with GSP axons $(P=0.007)$ and CT axons $(P=0.008)$. On average, $22.0 \pm 4.1$ synapses $/ \mathrm{mm}^{3}$ were found for IX axons vs. an average ( \pm SEM) of $4.4 \pm$ 0.8 synapses $/ \mathrm{mm}^{3}$ for GSP axons and an average of $4.9 \pm$ 0.9 synapses $/ \mathrm{mm}^{3}$ for CT axons (Fig. 8C). The difference in the volumetric density of synapses associated with GSP axons and with CT axons was not statistically significant $(P=0.9)$.

\section{DISCUSSION}

The results of this study reveal for the first time the morphological characteristics of identified GSP, CT, and IX afferent terminals in the rat NTS. In the zone that receives overlapping inputs from the three nerves, all nerves displayed similar characteristics in vesicular shape and asymmetry of synaptic contacts, which are typical of excitatory nerve terminals. However, there were notable differences in the inputs that the three nerves provide to the NTS. The ultrastructural characteristics of CT and GSP terminals were similar to each other and often differed from measures in IX terminals.

\section{Presynaptic terminal size and postsynaptic target differences}

One of the most salient differences among afferents of the three nerves was the dichotomy in presynaptic terminal areas. IX axons displayed larger terminal endings than GSP and CT axons, whereas GSP and CT axon terminals were similar in size. These results are consistent with those found in hamster by Whitehead (1986) and Brining and Smith (1996). Another prominent feature that distinguished IX terminations from GSP and CT terminations was the postsynaptic targets of these afferents. Synapses from GSP and CT axons occurred most frequently on dendritic shafts, whereas synapses on IX axons occurred more readily on dendritic spines.

It is not surprising that CT and GSP nerves have similarly sized terminals and share similar types of synaptic partners, insofar as both nerves are branches of the facial nerve and both have somas in the geniculate ganglia. Thus, the disparity of IX axon terminal size and target preference compared with CT and GSP axon terminals could be a reflection of the differences associated with the developmental histories and cellular characteristics of the two cranial nerves. Neurons comprising the CT and GSP in the geniculate ganglion are born on embryonic day 11 (E11), whereas petrosal ganglion (IXth nerve) cells are born at E14 (Altman and Bayer, 1982). The developmental difference in birth dates might also be reflected in their initial projection and subsequent elaboration within the NTS. As noted earlier, the mere presence of a labeled synapse from GSP, CT, or IX axons does not necessarily mean that it conveys taste information. All of these nerves also carry nongustatory (e.g., tactile) information to the NTS (Frank, 1991; Nejad, 1986; Ogawa et al., 1968; Sollars and Hill, 1998). Therefore, these findings provide information about anatomical features of afferent input to target cells in the NTS but await companion findings that assess the function of such inputs.

\section{Additional morphological differences}

An additional noticeable dissimilarity between the VII and the IX afferent terminals was the presence of dendritic spinules. These protrusions invaginated IX axon terminals markedly more often than GSP or CT axon terminals. Spinules, similar to those observed in this study, have been described in detail in other brain regions, including area CA1 of the hippocampus (Spacek and Har- 


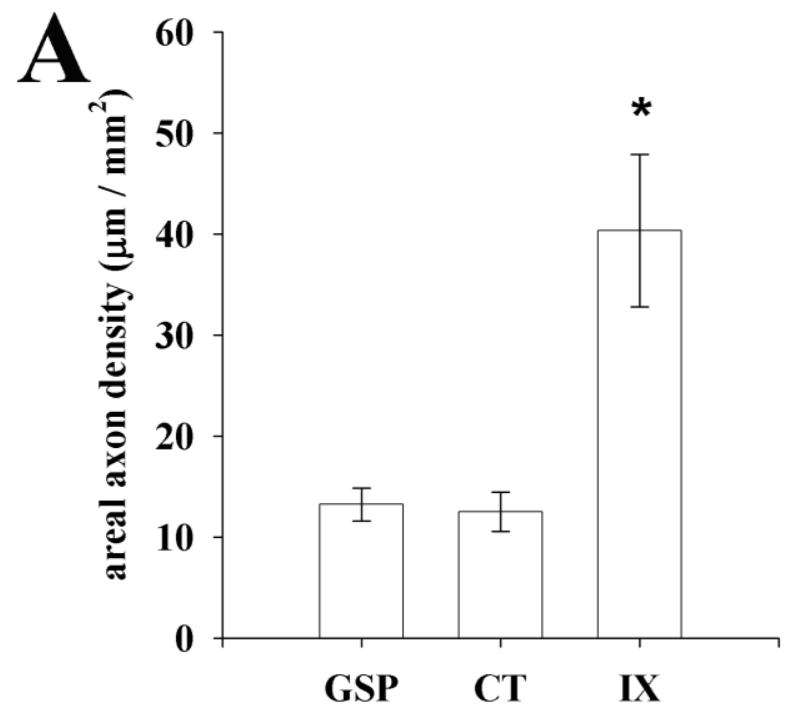

B
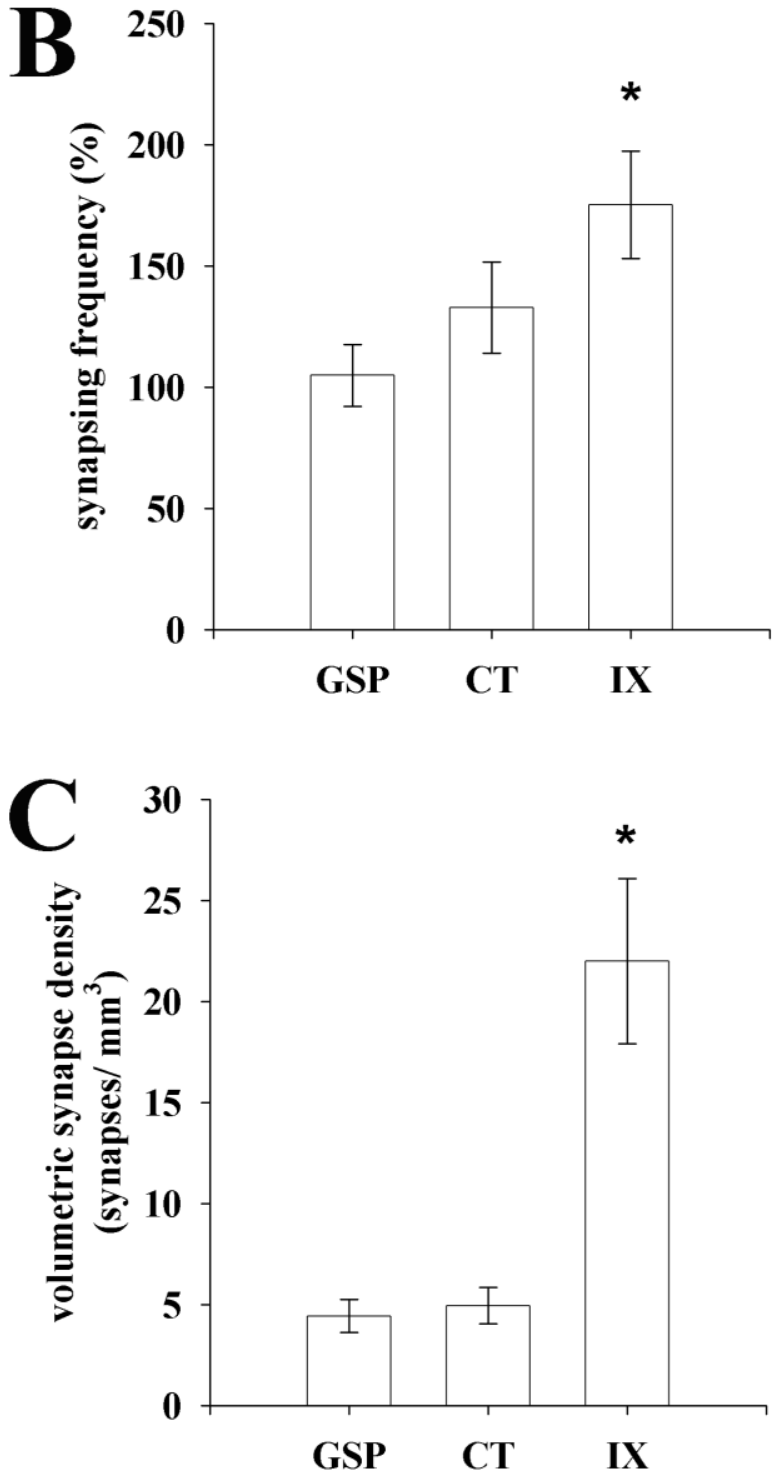
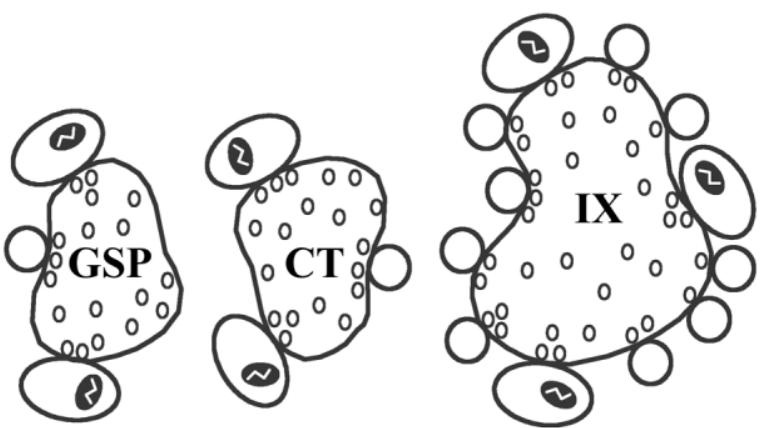

Fig. 9. Schematic of density and frequency distributions and dendrite/spine targeting of the axon terminals of the GSP, CT, and IX nerves. IX axon terminals are larger than GSP and CT axon terminals and synapse more often on dendritic spines (circles) than dendritic shafts (large ovals). Dendrites contain mitochondria (solid symbols). Small ovals inside of axon terminals represent synaptic vesicles. GSP and CT axon terminals synapse more often on dendritic shafts. IX axon terminals made the majority of synaptic contacts onto NTS neurons. For descriptive purposes, synapses represented by this model are meant to indicate the estimated synapse density and frequency of afferent terminals in the entire dorsal NTS.

ris, 2004), dentate gyrus of the hippocampus (Tarrant and Routtenberg, 1977), cerebellum (Eccles et al., 1967), frontal cortex (Cadete-Leite et al., 1986), sensorimotor cortex (Bozhilova-Pastirova and Ovtscharoff, 1999), and visual cortex (Erisir and Dreusicke, 2005), suggesting a commonality of afferent, excitatory input throughout the brain. Although the function of the spinule remains speculative (Spacek and Harris, 2004), the existence of these protrusions in the NTS neuropil increases the postsynaptic surface area available with which IX terminals can make synaptic contacts. Moreover, the presence of larger IX terminals having a majority of their synaptic input occurring on spines in conjunction with the presence of spinules elevates the likelihood for IX terminals to engulf their postsynaptic partners.

\section{Density and frequency differences}

The synaptic arrangements of each gustatory afferent in the dorsal NTS, quantified by synapse frequency and density measurements, also distinguished the axonal input of each nerve (Fig. 9). Our results reveal that, within the NTS region that we studied, where GSP, CT, and IX axons overlap, IX axons were by far the densest, and each IX axon made the most frequent synapses. Within a given volume of the dorsal NTS, synapses on IX axons were four

Fig. 8. A: Areal axon density of DAB-labeled GSP, CT, and IX axons per square millimeter of the dorsal NTS. IX axons were fourfold denser than GSP and CT axons located in the same plane. The densities of GSP and CT axons were not significantly different. B: Synapsing frequency of DAB-labeled GSP, CT, and IX axon terminals (see Results for interpretation). IX axons formed synapses at a significantly higher frequency than GSP axons but did not differ from CT axons. C: Volumetric density of synapses associated with DABlabeled GSP, CT, and IX axons. Four times as many synapses were present on IX axons as on GSP and CT axons. There was no significant difference in the density of synapses associated with GSP and CT axons. See Materials and Methods for calculations. Error bars indicate standard error. ${ }^{*} P<0.05$. 
times as dense as synapses on GSP and CT axons. Also, the estimated total density of IX axons was fourfold greater than that of both CT and GSP axons; CT and GSP axons terminated in the dorsal zone in similar proportions. Hence, IX axons occupied more volume in the dorsal zone of the NTS compared with CT and GSP axons, and the IX axon terminals engaged in more synaptic contacts with their postsynaptic targets (Fig. 9). Compared with the synaptic junctions on GSP and CT terminals, this disproportionate distribution of synaptic junctions on IX axon terminals is most likely related to physical capacity. The relatively large size of the IX terminal gives it more surface area with which to engage in synaptic contacts. Furthermore, the formation of synapses on spines by these large IX terminals increases the packing density of synaptic contacts, which would not be achieved by formation of synapses directly onto dendritic shafts. Both the size and the postsynaptic target of IX terminals enable these terminals to make more synaptic contacts with NTS neurons. Therefore, in comparing the density and distribution of synaptic contacts upon afferent terminals in this specific area of the NTS that appears especially plastic (King and Hill, 1991; Krimm and Hill, 1997; May and Hill, 2006; Sollars et al., 2006), the majority of synaptic junctions are associated with the IXth nerve. We must emphasize, however, that this distribution of synapses in the dorsal zone would not be sustained in more intermediate and ventral portions of the NTS (Fig. 1). The terminal field of the IXth nerve does not extend into these zones, whereas the projections from the GSP and CT are the most dense (May and Hill, 2006).

\section{Comparison of species}

The morphological properties of GSP, CT, and IX terminals characterized by these results are similar to the ultrastructure in hamster described by Whitehead (1986) and Brining and Smith (1996). However, the synapse frequency and density measurements of each individual nerve included in the current study were not examined previously, and it is unlikely that the area of neuropil sampled was the same between studies and similar to the area examined here. As in rat, hamster primary gustatory axons formed mostly axodendritic synapses that were asymmetric. In both species, synaptic endings were typically ovoid profiles, filled with round clear vesicles and abutted by surrounding processes that made synaptic connections. Hamster VII nerve terminals were on average $1.2 \mu \mathrm{m}$ in diameter and often engaged in complex glomerular-type arrangements involving both smallcaliber dendrites or spines and unlabeled terminals (Whitehead, 1986). IXth nerve terminals were larger, with an average $1.8 \mu \mathrm{m}$ diameter, and engaged in simpler synaptic junctions, most often with dendritic spines (Brining and Smith, 1996). We found similar size differences between VII and IX terminals in rat and observed both simple and glomerular arrangements of these terminals. In fact, the morphological characteristics of the IXth nerve terminals of rat appeared similar to those of hamster. The results of this study provide additional evidence that the components of the rat VII (CT and GSP) are similar to those reported for the hamster VII.

\section{Potential for synaptic convergence}

GSP, CT, and IX nerves all terminate in an overlapping pattern on the rostral NTS in the zone of the NTS upon which this study focused (May and Hill, 2006). This portion of the NTS also receives overlapping projections from the lingual branch of the trigeminal nerve (V), which provides somatosensory innervation (Hamilton and Norgren, 1984). Thus, there is a potential for convergence of these inputs onto the same postsynaptic cells. Indeed, electrophysiological studies show that single NTS neurons are responsive to taste stimuli applied to different receptor populations throughout the oral cavity (Sweazey and Smith, 1987; Travers et al., 1986). Our results that characterize the morphology and synaptology of individual nerve terminals provide the anatomical basis for further studies aimed at understanding how sensory inputs are integrated in the NTS and may allow for insights into why this area is particularly susceptible to developmentally and environmentally induced changes.

\section{ACKNOWLEDGMENTS}

We extend our appreciation to Ms. Bonnie L. Sheppard for her technical assistance with ultramicrotomy.

\section{LITERATURE CITED}

Altman J, Bayer S. 1982. Development of the cranial nerve ganglia and related nuclei in the rat. Adv Anat Embryol Cell Biol 74:1-90.

Bozhilova-Pastirova A, Ovtscharoff W. 1999. Intramembranous structure of synaptic membranes with special reference to spinules in the rat sensorimotor cortex. Eur J Neurosci 11:1843-1846.

Bradley RM, editor. 2007. The role of the nucleus of the solitary tract in gustatory processing. New York: CRC, Taylor and Francis.

Bradley RM, Grabauskas G. 1998. Neural circuits for taste. Excitation, inhibition, and synaptic plasticity in the rostral gustatory zone of the nucleus of the solitary tract. Ann N Y Acad Sci 855:467-474.

Brining SK, Smith DV. 1996. Distribution and synaptology of glossopharyngeal afferent nerve terminals in the nucleus of the solitary tract of the hamster. J Comp Neurol 365:556-574.

Cadete-Leite A, Tavares MA, Paula-Barbosa MM, Gray EG. 1986. "Perforated" synapses in frontal cortex of chronic alcohol-fed rats. J Submicrosc Cytol 18:495-499.

Cheon BK, Hill DL. 2003. Nerve cut induced decrease of chorda tympani terminal fields in the NTS of adult control and sodium-restricted rats. Chem Senses 28:557.

Colonnier M. 1968. Synaptic patterns on different cell types in the different laminae of the cat visual cortex. An electron microscope study. Brain Res 9:268-287.

Colonnier M, Beaulieu C. 1985. An empirical assessment of stereological formulae applied to the counting of synaptic disks in the cerebral cortex. J Comp Neurol 231:175-179.

Contreras RJ, Beckstead RM, Norgren R. 1982. The central projections of the trigeminal, facial, glossopharyngeal and vagus nerves: an autoradiographic study in the rat. J Auton Nerv Syst 6:303-322.

Davis BJ. 1998. Synaptic relationships between the chorda tympani and tyrosine hydroxylase-immunoreactive dendritic processes in the gustatory zone of the nucleus of the solitary tract in the hamster. J Comp Neurol 392:78-91.

DeFelipe J, Marco P, Busturia I, Merchan-Perez A. 1999. Estimation of the number of synapses in the cerebral cortex: methodological considerations. Cereb Cortex 9:722-732.

Eccles JC, Ito MSJ, Szentagothai J. 1967. The cerebellum as a neuronal machine. New York: Springer. p 127-130.

Erb DE, Povlishock JT. 1991. Neuroplasticity following traumatic brain injury: a study of GABAergic terminal loss and recovery in the cat dorsal lateral vestibular nucleus. Exp Brain Res 83:253-267.

Erickson RP. 1966. Nontraumatic headholder for rats. Physiol Behav 1:9798.

Erisir A, Dreusicke M. 2005. Quantitative morphology and postsynaptic targets of thalamocortical axons in critical period and adult ferret visual cortex. J Comp Neurol 485:11-31.

Frank ME. 1991. Taste-responsive neurons of the glossopharyngeal nerve of the rat. J Neurophysiol 65:1452-1463. 
Gillingwater TH, Ingham CA, Parry KE, Wright AK, Haley JE, Wishart TM, Arbuthnott GW, Ribchester RR. 2006. Delayed synaptic degeneration in the CNS of Wlds mice after cortical lesion. Brain 129:15461556.

Gould E, Woolley CS, Frankfurt M, McEwen BS. 1990. Gonadal steroids regulate dendritic spine density in hippocampal pyramidal cells in adulthood. J Neurosci 10:1286.

Guillery RW. 2002. On counting and counting errors. J Comp Neurol 447:1-7.

Guillery RW, August BK. 2002. Doubt and certainty in counting. Prog Brain Res 135:25-42.

Halsell CB, Travers SP, Travers JB. 1996. Ascending and descending projections from the rostral nucleus of the solitary tract originate from separate neuronal populations. Neuroscience 72:185-197.

Hamilton RB, Norgren R. 1984. Central projections of gustatory nerves in the rat. J Comp Neurol 222:560-577.

Hayat MA. 2000. Principles and techniques of electron microscopy. Biological applications. New York: Cambridge University Press.

Hubel DH, Wiesel TN, LeVay S. 1977. Plasticity of ocular dominance columns in monkey striate cortex. Philos Trans R Soc Lond B Biol Sci 278:377-409.

Killackey HP, Ivy GO, Cunningham TJ. 1978. Anomalous organization of SMI somatotopic map consequent to vibrissae removal in the newborn rat. Brain Res 155:136-140.

King CT, Hill DL. 1991. Dietary sodium chloride deprivation throughout development selectively influences the terminal field organization of gustatory afferent fibers projecting to the rat nucleus of the solitary tract. J Comp Neurol 303:159-169.

King CT, Hill DL. 1993. Neuroanatomical alterations in the rat nucleus of the solitary tract following early maternal $\mathrm{NaCl}$ deprivation and subsequent NaCl repletion. J Comp Neurol 333:531-542.

Krimm RF, Hill DL. 1997. Early prenatal critical period for chorda tym pani nerve terminal field development. J Comp Neurol 378:254-264.

Lasiter PS. 1992. Postnatal development of gustatory recipient zones within the nucleus of the solitary tract. Brain Res Bull 28:667-677.

Lasiter PS, Kachele DL. 1990. Effects of early postnatal receptor damage on development of gustatory recipient zones within the nucleus of the solitary tract. Brain Res Dev Brain Res 55:57-71.

Lasiter PS, Wong DM, Kachele DL. 1989. Postnatal development of the rostral solitary nucleus in rat: dendritic morphology and mitochondrial enzyme activity. Brain Res Bull 22:313-321.

Loewy AD, Burton H. 1978. Nuclei of the solitary tract: efferent projections to the lower brain stem and spinal cord of the cat. J Comp Neurol 181:421-449.

Mangold JE, Hill DL. 2004. Gustatory terminal fields in rats recovered from early developmental sodium restriction. Proc ISOT/JASTS Abstr 115

Mangold JE, Hill DL. 2005. Postnatal development of gustatory nerve terminal fields in control rats. Chem Senses 30:A166.

May OL, Hill DL. 2006. Gustatory terminal field organization and developmental plasticity in the nucleus of the solitary tract revealed through triple-fluorescence labeling. J Comp Neurol 497:658-669.

Nejad MS. 1986. The neural activities of the greater superficial petrosal nerve of the rat in response to chemical stimulation of the palate. Chem Senses 11:283-293.

Ogawa H, Sato M, Yamashita S. 1968. Multiple sensitivity of chorda tympani fibres of the rat and hamster to gustatory and thermal stimuli. J Physiol 199:223-240.

Peters A, Palay SL, Webster Hd. 1970. Fine structure of the nervous system. New York: Harper and Row.
Pierce JP, Lewin GR. 1994. An ultrastructural size principle. Neuroscience 58:441-446.

Pittman DW, Contreras R. 2002. Dietary NaCl influences the organization of chorda tympani neurons projecting to the nucleus of the solitary tract in rats. Chem Senses 27:333-341.

Smith DV, Li CS. 2000. GABA-mediated corticofugal inhibition of tasteresponsive neurons in the nucleus of the solitary tract. Brain Res 858:408-415.

Smith DV, Scott TR. 2003. Gustatory neural coding. In: Doty RL, editor. Handbook of olfaction and gustation. New York: Marcel Dekker. p 731-758.

Sollars SI, Hill DL. 1998. Taste responses in the greater superficial petrosal nerve: substantial sodium salt and amiloride sensitivities demonstrated in two rat strains. Behav Neurosci 112:991-1000.

Sollars SI, Hill DL. 2000. Lack of functional and morphological susceptibility of the greater superficial petrosal nerve to developmental dietary sodium restriction. Chem Senses 25:719-727.

Sollars SI, Walker BR, Thaw AK, Hill DL. 2006. Age-related decrease of the chorda tympani nerve terminal field in the nucleus of the solitary tract is prevented by dietary sodium restriction during development. Neuroscience 137:1229-1236.

Somogyi P, Bolam JP, Smith AD. 1981. Monosynaptic cortical input and local axon collaterals of identified striatonigral neurons. A light and electron microscopic study using the Golgi-peroxidase transportdegeneration procedure. J Comp Neurol 195:567-584

Spacek J, Harris KM. 2004. Trans-endocytosis via spinules in adult rat hippocampus. J Neurosci 24:4233-4241.

Sweazey RD, Smith DV. 1987. Convergence onto hamster medullary taste neurons. Brain Res 408:173-184.

Tarrant SB, Routtenberg A. 1977. The synaptic spinule in the dendritic spine: electron microscopic study of the hippocampal dentate gyrus. Tissue Cell 9:461-473.

Travers SP, Pfaffmann C, Norgren R. 1986. Convergence of lingual and palatal gustatory neural activity in the nucleus of the solitary tract. Brain Res 365:305-320.

Waite PM, Cragg BG. 1982. The peripheral and central changes resulting from cutting or crushing the afferent nerve supply to the whiskers. Proc R Soc Lond B Biol Sci 214:191-211.

Whitehead MC. 1986. Anatomy of the gustatory system in the hamster: synaptology of facial afferent terminals in the solitary nucleus. J Comp Neurol 244:72-85.

Whitehead MC. 1988. Neuronal architecture of the nucleus of the solitary tract in the hamster. J Comp Neurol 276:547-572.

Whitehead MC. 1993. Distribution of synapses on identified cell types in a gustatory subdivision of the nucleus of the solitary tract. J Comp Neurol 332:326-340.

Wiesel TN, Hubel DH. 1965. Comparison of the effects of unilateral and bilateral eye closure on cortical unit responses in kittens. J Neurophysiol 28:1029-1040.

Woolley CS, Wenzel HJ, Schwartzkroin PA. 1996. Estradiol increases the frequency of multiple synapse boutons in the hippocampal CA1 region of the adult female rat. J Comp Neurol 373:108.

Woolley CS, Weiland NG, McEwen BS, Schwartzkroin PA. 1997. Estradiol increases the sensitivity of hippocampal CA1 pyramidal cells to NMDA receptor-mediated synaptic input: correlation with dendritic spine density. J Neurosci 17:1848.

Zhang LL, Ashwell KW. 2001. The development of cranial nerve and visceral afferents to the nucleus of the solitary tract in the rat. Anat Embryol 204:135-151. 